\title{
PREVALENCE OF AGE-RELATED MACULOPATHY AT TWO POINTS IN TIME IN AN ELDERLY BRITISH POPULATION
}

\author{
A. J. DICKINSON ${ }^{1}$, J. M. SPARROW ${ }^{2}$, A. M. DUKE ${ }^{2}$, J. R. THOMPSON ${ }^{3}$, J. M. GIBSON ${ }^{4}$ and \\ A. R. ROSENTHAL ${ }^{3}$ \\ Newcastle, Bristol, Leicester and Birmingham
}

\begin{abstract}
SUMMARY
As the demography of Western society changes, the population prevalence of diseases such as age-related macular degeneration (AMD) is expected to rise. Despite this, there remains a paucity of quality data concerning the population prevalence of $A M D$, the commonest cause of blindness in the elderly.

Purpose: To report the prevalence of AMD at two points in time in an elderly population.

Method: A geographically defined random population sample of elderly people was defined in 1980, and studied in 1982-4. In 1990, a cohort of survivors was identified. Participants underwent full ophthalmic examination with fundus photography using the same camera on each occasion. Photographs were randomly encoded and graded by two independent masked observers using the Wisconsin Age-related Maculopathy Grading System. Disagreements were resolved by consensus.
\end{abstract}

Results: Eighty-eight survivors participated in the follow-up examinations. Of these, 82 subjects had gradable retinal photographs for both examination points in at least one eye. There were 158 pairs of images (initial and subsequent) available for analysis. The mean age was 80 years (range 77-90 years) at the initial examination, and 87 years (range 84-97 years) at the subsequent examination; $70.7 \%$ of subjects were female. Prevalence rates for the initial examination were: drusen $72.8 \%$, drusen confluence $37.3 \%$, degeneration of the retinal pigment epithelium (RPE) $51.3 \%$, increased pigment $22.2 \%$, exudative AMD $1.9 \%$ and

From: ${ }^{1}$ Department of Ophthalmology, Royal Victoria Infirmary, Newcastle upon Tyne; ${ }^{2}$ University Department of Ophthalmology, Bristol Eye Hospital, Bristol; ${ }^{3}$ University Department of Ophthalmology, Leicester Royal Infirmary, Leicester, ${ }^{4}$ Department of Ophthalmology, Birmingham Heartlands Hospital, Birmingham, UK.

Correspondence to: $\mathrm{Mr}$ J. M. Sparrow, DPhil, FRCS, FRCOphth, University Department of Ophthalmology, Bristol Eye Hospital, Bristol BS1 2LX, UK. Fax: +44 (117) 9251421. geographic atrophy $1.9 \%$. Rates at second examination were: drusen $62.7 \%$ drusen confluence $41.8 \%$, RPE degeneration $72.8 \%$, increased pigment $16.5 \%$, exudative AMD 3.8\% and geographic atrophy $3.2 \%$.

Conclusion: This 'double' prevalence study provides detailed data on AMD lesions at two points in time in a population-based group of elderly people.

The demography of Western nations is changing, and by the end of the century, $6.5 \%$ of the European Union will be aged 75 years or over. ${ }^{1}$ Hence there is a pressing need for more information on the epidemiology of common age-related problems if we are to anticipate the health care requirements of the coming decades.

There is little detailed and representative information on age-related macular degeneration (AMD) from populations over 75 years of age ${ }^{2-6}$ and data are extremely scarce on persons over 85 years, who in the United Kingdom alone now total almost 850000 . $^{7}$ Epidemiological documentation of AMD has also been hampered by the lack of universally accepted disease criteria. ${ }^{8-10}$ Some authors have defined AMD using histopathological criteria such as the presence of a continuous layer of basal laminar deposit under the macula, ${ }^{11,12}$ whilst others have defined it in a variety of clinical ways, one of the most frequent being the presence of any AMD feature associated with a visual acuity of $6 / 9$ or worse. ${ }^{13,14}$ Similarly, methods of disease quantification vary widely. ${ }^{15}$ For example, post-mortem studies on persons over 40 years have shown prevalence rates for macular drusen to vary from $37 \%$ to $87 \%$, ${ }^{16,17}$ whereas clinical studies have defined rates between $18.4 \%$ and $>92 \%$ for persons over 60 years old. ${ }^{5,6}$

The purpose of this study was to document, at two points in time, the prevalence rates for detailed features of AMD in a population-based sample of elderly individuals. The sample we chose for study 
was the survivors of a cohort of elderly individuals who had originally been examined between 1982-4 by Gibson et al. ${ }^{4}$ Incidence data and the natural history of AMD lesions in this population are presented in a separate report. ${ }^{18}$

There is now good evidence that drusen alter in size and morphology with time. ${ }^{12,19,20}$ In addition, certain types of drusen may be associated with an increased risk of exudative AMD. ${ }^{12,21,22}$ Hence very detailed systems of description and quantification with acceptable repeatability are essential to the understanding of AMD. ${ }^{23}$ The Wisconsin Agerelated Maculopathy Grading System is one such system. ${ }^{24,25}$

\section{METHOD}

The study was conducted in Melton Mowbray, Leicestershire, an English market town with a population of 32000 persons. It is served by a single general practice, whose age-sex register is numerically virtually identical to the population of the town and surrounding rural area. In 1981, the Department of Epidemiology and Public Health of the University of Leicester carried out a comprehensive survey of all persons on the register aged 75 years and older. ${ }^{26}$ From this population of over 1300 , they achieved a response rate in excess of $90 \%$, and a random sample from this group formed the basis of an eye study. Between 1982 and 1984 a single ophthalmologist and an optometrist carried out full mydriatic ophthalmic examinations on 529 participants, who represented $71.5 \%$ of the targeted sample. ${ }^{4}$ During the examination, $30^{\circ}$ macular colour transparencies were taken using a non-stereoscopic Zeiss Jena fundus camera.

In 1990, the resident survivors from this cohort were identified and invited to attend for a further ophthalmic examination. Eligible subjects were contacted by post and offered an appointment to attend the research clinic. This initial approach was backed up with a telephone call to explain the main aims of the research and to offer transport if this was required. At the research clinic a medical and ophthalmic history was obtained from participants, who then underwent a full ocular examination. This took place in the same hospital clinic as before. Examinations were performed by one of the two study ophthalmologists and by the same optometrists. Fundus photography with the same retinal camera produced a second set of $30^{\circ}$ macular colour transparencies for masked comparison with the photographs of 7 years previously.

The slide transparencies were mounted, and a grid, centred on the fovea, was superimposed in accordance with the protocol of the Wisconsin Agerelated Maculopathy Grading System. ${ }^{24}$ This grid consisted of three concentric circles and four radial lines arranged to define nine subfields: one central, four inner and four outer. The radius of the innermost circle corresponded to $500 \mu \mathrm{m}$ of an average fundus, and the radii of the middle and outer circles to 1500 and $3000 \mu \mathrm{m}$ respectively. Three sets of smaller circles printed on transparent plastic allowed estimation of size and area of drusen and other features. A $\times 5$ magnification monocular slide viewer and a standard desk-mounted fluorescent viewing box of unknown Kelvin rating were used. Grading of all macular photographs was performed by two independent masked observers, with sequential grading of right and left eyes. In no instances were the images from initial and subsequent examinations of the same eye graded on the same day, the time interval in most cases being more than a month. All grading disagreements were resolved by review to reach a consensus between the two observers, who remained masked until the completion of this process.

The Wisconsin system was used to grade the following drusen features in each subfield: maximum drusen size, predominant drusen type (those occupying the largest area) and drusen area. Predominant (most common) drusen size and drusen confluence (the largest diameter of two or more touching drusen) were graded for all nine subfields together. From the areas of the subfields and the drusen area scores, a calculation of the total drusen area for the eye as a whole was made. Degeneration of the retinal pigment epithelium (RPE), increased pigment, subretinal haemorrhage, fibrous scarring and geographic atrophy were also graded by subfield. The lack of stereoscopic photographs necessitated a number of minor modifications to the Wisconsin protocol. Where the definition of drusen type relied on stereoscopic features such as 'solid, thick and nodular', we chose to re-define drusen as being 'hard' when of $<63 \mu \mathrm{m}$ in diameter and 'soft' when $\geqslant 63 \mu \mathrm{m}$. The grading of 'elevated' features such as RPE detachments was omitted. Image quality was defined as 'good' where retinal detail was unimpaired, 'fair' where retinal detail was slightly impaired but distinct drusen of $63 \mu \mathrm{m}$ would still be visible, and 'poor' where such drusen were deemed not to be visible. Pigmentary disturbance in the Wisconsin system is divided into RPE degeneration or hypo-pigmentation (which falls short of classical geographic atrophy), and hyper-pigmentation which is termed increased pigmentation. Where minor points of detail were not covered adequately by the Wisconsin protocol, these were defined locally and followed throughout. Rigid adherence to the Wisconsin protocol was otherwise maintained.

Inter-observer and intra-observer variability were analysed by weighted and unweighted kappa statistics and percentage agreement scores. Agreement levels were moderate to substantial for most features. ${ }^{25}$ 


\section{Age Distributions}

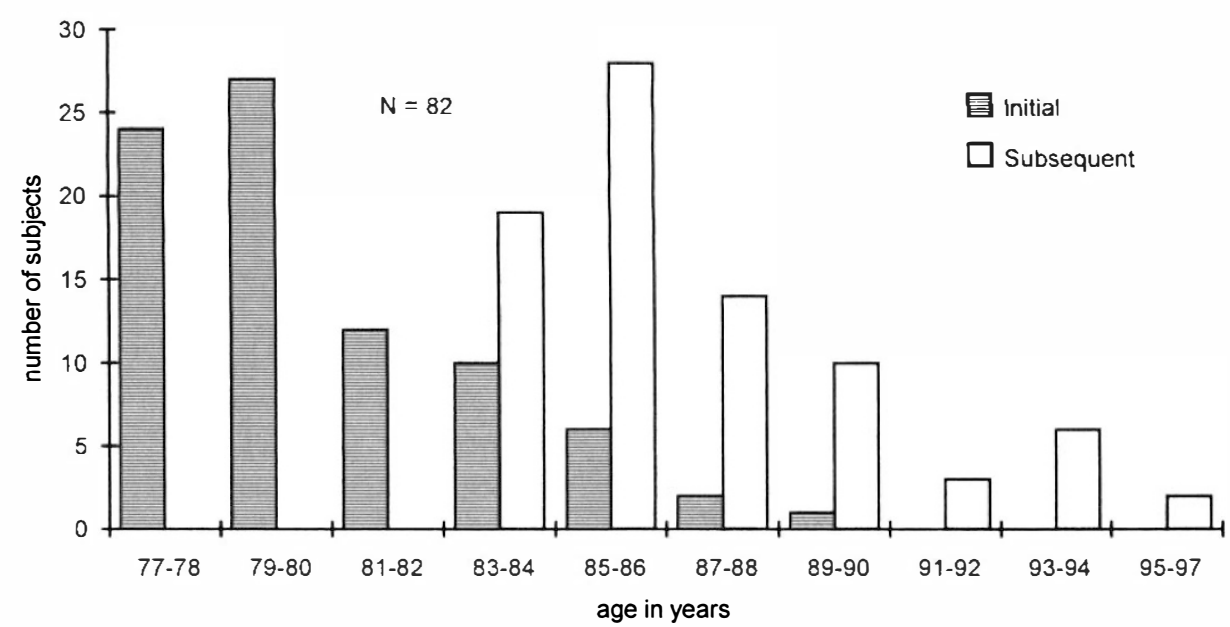

Fig. 1. Age distributions at initial and subsequent assessments of participants with gradable photographs of at least one eye. Mean age at initial examination was 80 years (range 77-90 years) and at subsequent examination was 87 years (range 84-97 years).

The study was approved by the ethics committee with responsibility for research in the Leicester group of hospitals and all subjects gave informed verbal consent prior to participation.

\section{RESULTS}

At the time of the second examination survivorship information was available for $99.5 \%$ (all except 3 individuals) of those who participated in the original eye study. Known survivors numbered 223, of whom 19 had moved, 20 could not be contacted at their former address and 8 were too unwell to attend. Of the remaining 176 resident survivors, $88(50.0 \%)$ participated in the study, of whom $70.7 \%$ were female.

As there was only a $50 \%$ response rate at the subsequent examination, comparisons were made between known survivors who attended and survivors who did not attend the subsequent examination. Using data from the earlier study, there were no age or sex differences between the attenders and the nonattenders $(p>0.26$ for both, by $t$-test and chi-square respectively), suggesting that the attenders were demographically representative of the surviving population as a whole. Best corrected visual acuity (as recorded in the earlier eye study for the better eye) was also compared for attenders and nonattenders. Eighty-six per cent of attenders had an acuity of $6 / 12$ or better, while only $76 \%$ of nonattenders achieved this level of vision at baseline. This difference was, however, not significant by chi-square testing $(p=0.10)$, indicating that although attenders appeared at baseline to be slightly under-representative of visual morbidity, the differences in acuity between attenders and non-attenders were within expectations of chance variation due to sampling.

Photographs were deemed gradable when at least one feature could be graded according to the Wisconsin protocol. When this was not the case it was most often due to media opacities. Of the 88 attenders, 82 ( $47 \%$ of 176 survivors) had gradable photographs of at least one eye, allowing the final analysis to be made on 158 eyes with gradable photographs from both examinations. In Fig. 1 the age distributions at both points in time are shown for the 82 participants with gradable images. The mean age of these individuals at initial assessment was 80

Table I. Ocular abnormalities at initial and subsequent assessments

\begin{tabular}{|c|c|c|c|c|c|}
\hline$n=158$ & Aphakia & $\begin{array}{l}\text { AMD (VA 6/18 } \\
\text { or worse) }\end{array}$ & $\begin{array}{c}\text { Cataract (VA } 6 / 18 \\
\text { or worse) }\end{array}$ & $\begin{array}{l}\text { Other }^{\mathrm{a}} \text { (VA } 6 / 18 \\
\text { or worse) }\end{array}$ & $\begin{array}{l}\text { All causes (VA } \\
6 / 18 \text { or worse) }\end{array}$ \\
\hline $\begin{array}{l}\text { Initial } \quad(n) \\
\text { \% of the eyes with } 6 / 18 \text { or worse }\end{array}$ & $\begin{array}{c}1 \\
0.6\end{array}$ & $\begin{array}{c}23 \\
14.6 \\
50.0\end{array}$ & $\begin{array}{c}15 \\
9.5 \\
32.6\end{array}$ & $\begin{array}{c}8 \\
5.1 \\
17.4\end{array}$ & $\begin{array}{r}46 \\
29.1 \\
100\end{array}$ \\
\hline $\begin{array}{l}\text { Subsequent }(n) \\
\qquad(\%) \\
\% \text { of the eyes with } 6 / 18 \text { or worse }\end{array}$ & $\begin{array}{l}12 \\
7.6\end{array}$ & $\begin{array}{c}38 \\
24.1 \\
48.7\end{array}$ & $\begin{array}{c}35 \\
22.2 \\
44.9\end{array}$ & $\begin{array}{c}12 \\
7.6 \\
15.4\end{array}$ & $\begin{array}{r}78 \\
49.4 \\
100\end{array}$ \\
\hline
\end{tabular}

Data represent 'rough and ready' clinical assessments in the research clinic. Examining ophthalmologists were asked to assign a cause for any observed reduction in acuity. The 158 eyes represented are those for which retinal photographic grades later became available for both initial and subsequent occasions. Some eyes had features of more than one pathology (e.g. combined cataract and AMD).

"Other visually significant pathologies included one or more of the following: retinal vein occlusion, diabetic retinopathy, 'optic atrophy' of uncertain cause - presumed ischaemic, glaucoma, myopic degeneration, and cellophane maculopathy. 
years (range 77-90 years), and at the subsequent assessment was 87 years (range 84-97 years).

Information derived from the research clinic assessments of the 158 eyes is presented in Table I. These data were collected with the participants in attendance and do not contain the same level of detail as that from the grading of the retinal photographs. The overall numbers of eyes with acuity of $6 / 18$ or worse due to AMD, cataract and 'other' causes were each higher at the subsequent examination. In addition, of the affected eyes, there were proportionally slightly more with cataract subsequently, although the proportions affected by AMD and 'other' pathologies were similar on the two occasions.

From the grading of the retinal images detailed summary information on AMD features at both initial and subsequent examinations is provided in Tables II-IX (data are presented in a 'by eye' format). Where frequencies permitted, subjects were subdivided into three age bands according to age at subsequent examination: $84-85$ years (65 eyes), 86-88 years (54 eyes) and 89-97 years (39 eyes). These divisions were chosen arbitrarily to provide sufficient numbers to allow comparison between age bands. To facilitate a more direct comparison between initial and subsequent observations, the same individuals were grouped into approximate age bands at initial examination. These age bands (77-78 years, 79-81 years and 82-90 years) were approximate because the follow-up period varied slightly between subjects. For certain features, subfield analyses for 'central and inner' and 'central only' are presented to highlight specific points of interest.

\section{Drusen}

At initial examination, definite drusen (Table II, grade 2 or more) were present in the macula of 115 of 158 eyes $(72.8 \%)$. The frequency of definite drusen was lower at the subsequent examination 99 of $158(62.7 \%)$ of eyes - and this coincided with an increased number of photographs where the quality was too poor to allow drusen grading, generally due to lens opacities. After exclusion of images which were ungradable for drusen, the frequencies were more similar at $74 \%$ and $67 \%$ respectively.

The maximum drusen size per eye on examination of all subfields is shown in Table II. At the initial examination distinct drusen in the size ranges $<63 \mu \mathrm{m}$ (grade 2) and 63 to $<125 \mu \mathrm{m}$ (grade 3) occurred with similar frequencies $(24.7 \%$ and $26.0 \%$, respectively). At the subsequent examination, however, reticular drusen (grade 6) were the commonest maximum-sized drusen, being present in $25.3 \%$ of eyes. On each occasion the oldest subgroup of individuals had the highest prevalence of reticular drusen. The predominant (commonest as opposed to maximum) drusen size present in the grid area as a whole is shown in Table III. Similarly, small hard drusen (grade 2) predominated at the initial examination, and reticular drusen (grade 6) predominated at the subsequent examination.

Table IV catalogues the prevalence of drusen type, determined as follows. The score for an eye was based on the assumption of a rank-ordered grading which proceeded: hard indistinct or questionable drusen or stippling (drusen doubtful); hard distinct drusen; soft distinct drusen; soft indistinct drusen; reticular drusen. The eye was rated according to the 'highest' grade occurring in any subfield, with subfields having been individually classified by the drusen type occupying the largest area of that subfield. Because there were very few soft indistinct

Table II. Maximum drusen size (per eye) for the three age groups at initial and subsequent assessments

\begin{tabular}{|c|c|c|c|c|c|c|c|c|c|}
\hline \multirow{2}{*}{$\begin{array}{l}\text { Age at subsequent } \\
\text { examination }\end{array}$} & & \multicolumn{8}{|c|}{ Grade of maximum drusen size } \\
\hline & & 0 & 1 & 2 & 3 & 4 & 5 & 6 & $7 \& 8$ \\
\hline $\begin{array}{l}84-85 \text { years } \\
(n=65)\end{array}$ & $\begin{array}{l}\text { Initial } \\
\text { (\%) } \\
\text { Subsequent } \\
(\%)\end{array}$ & $\begin{array}{c}4 \\
6.2 \\
9 \\
13.9\end{array}$ & $\begin{array}{c}11 \\
16.9 \\
7 \\
10.8\end{array}$ & $\begin{array}{c}15 \\
23.1 \\
5 \\
7.7\end{array}$ & $\begin{array}{c}19 \\
29.2 \\
16 \\
24.7\end{array}$ & $\begin{array}{c}7 \\
10.8 \\
9 \\
13.9\end{array}$ & $\begin{array}{c}0 \\
0.0 \\
1 \\
1.5\end{array}$ & $\begin{array}{c}8 \\
12.3 \\
15 \\
23.1\end{array}$ & $\begin{array}{c}1 \\
1.5 \\
3 \\
4.6\end{array}$ \\
\hline $\begin{array}{l}86-88 \text { years } \\
(n=54)\end{array}$ & $\begin{array}{l}\text { Initial } \\
\text { (\%) } \\
\text { Subsequent } \\
(\%)\end{array}$ & $\begin{array}{c}9 \\
16.7 \\
14 \\
25.9\end{array}$ & $\begin{array}{c}7 \\
13.0 \\
7 \\
13.0\end{array}$ & $\begin{array}{c}17 \\
31.5 \\
7 \\
13.0\end{array}$ & $\begin{array}{c}13 \\
24.1 \\
9 \\
16.7\end{array}$ & $\begin{array}{c}4 \\
7.4 \\
2 \\
3.7\end{array}$ & $\begin{array}{c}0 \\
0.0 \\
0 \\
0.0\end{array}$ & $\begin{array}{c}4 \\
7.4 \\
12 \\
22.2\end{array}$ & $\begin{array}{c}0 \\
0.0 \\
3 \\
5.6\end{array}$ \\
\hline $\begin{array}{l}89-97 \text { years } \\
(n=39)\end{array}$ & $\begin{array}{l}\text { Initial } \\
\text { (\%) } \\
\text { Subsequent } \\
(\%)\end{array}$ & $\begin{array}{c}6 \\
15.4 \\
6 \\
15.4\end{array}$ & $\begin{array}{c}4 \\
10.3 \\
5 \\
12.8\end{array}$ & $\begin{array}{c}7 \\
18.0 \\
3 \\
7.7\end{array}$ & $\begin{array}{c}9 \\
23.1 \\
4 \\
10.3\end{array}$ & $\begin{array}{c}5 \\
12.9 \\
3 \\
7.7\end{array}$ & $\begin{array}{c}0 \\
0.0 \\
0 \\
0.0\end{array}$ & $\begin{array}{c}7 \\
18.0 \\
13 \\
33.3\end{array}$ & $\begin{array}{c}1 \\
2.6 \\
5 \\
12.8\end{array}$ \\
\hline $\begin{array}{l}\text { All } \\
(n=158)\end{array}$ & $\begin{array}{l}\text { Initial } \\
\text { (\%) } \\
\text { Subsequent } \\
\text { (\%) }\end{array}$ & $\begin{array}{c}19 \\
12.0 \\
29 \\
18.4\end{array}$ & $\begin{array}{c}22 \\
13.9 \\
19 \\
12.0\end{array}$ & $\begin{array}{c}39 \\
24.7 \\
15 \\
9.5\end{array}$ & $\begin{array}{c}41 \\
26.0 \\
29 \\
18.4\end{array}$ & $\begin{array}{c}16 \\
10.1 \\
14 \\
8.9\end{array}$ & $\begin{array}{c}0 \\
0.0 \\
1 \\
0.6\end{array}$ & $\begin{array}{c}19 \\
12.0 \\
40 \\
25.3\end{array}$ & $\begin{array}{c}2 \\
1.3 \\
11 \\
7.0\end{array}$ \\
\hline
\end{tabular}

Grade: 0 , no drusen; 1 , drusen hard indistinct, questionable or stippling only (drusen doubtful); 2 , hard distinct drusen, diameter $\leqslant 63 \mu \mathrm{m}$; 3 , drusen diameter $>6.3 \mu \mathrm{m}$ and $\leqslant 125 \mu \mathrm{m} ; 4$, drusen diameter $>125 \mu \mathrm{m}$ and $\leqslant 250 \mu \mathrm{m} ; 5$, drusen diameter $>250 \mu \mathrm{m} ; 6$, reticular drusen; $7 \& 8$, cannot grade (obscuring lesion and photograph quality amalgamated). Drusen size score for the eye as a whole was taken from the highest-scoring subfield. 
Table III. Predominant drusen size (per eye) for the three age groups at initial and subsequent assessments

\begin{tabular}{|c|c|c|c|c|c|c|c|c|c|c|}
\hline \multirow{2}{*}{$\begin{array}{l}\text { Age at subsequent } \\
\text { examination }\end{array}$} & & \multicolumn{9}{|c|}{ Grade of predominant drusen size } \\
\hline & & 0 & 1 & 2 & 3 & 4 & 5 & 6 & $7 \& 8$ & NA \\
\hline $\begin{array}{l}84-85 \text { years } \\
(n=65)\end{array}$ & $\begin{array}{l}\text { Initial } \\
(\%) \\
\text { Subsequent } \\
(\%) \\
\text { Initial } \\
\text { (\%) } \\
\text { Subsequent } \\
\text { (\%) }\end{array}$ & $\begin{array}{c}12 \\
18.5 \\
4 \\
6.2 \\
12 \\
22.2 \\
6 \\
11.1\end{array}$ & $\begin{array}{c}14 \\
21.5 \\
11 \\
16.9 \\
11 \\
20.4 \\
6 \\
11.1\end{array}$ & $\begin{array}{c}17 \\
26.2 \\
12 \\
18.5 \\
11 \\
20.4 \\
7 \\
13.0\end{array}$ & $\begin{array}{c}9 \\
13.9 \\
11 \\
16.9 \\
7 \\
13.0 \\
10 \\
18.5\end{array}$ & $\begin{array}{c}1 \\
1.5 \\
2 \\
3.1 \\
0 \\
0.0 \\
0 \\
0.0\end{array}$ & $\begin{array}{c}0 \\
0.0 \\
0 \\
0.0 \\
0 \\
0.0 \\
0 \\
0.0\end{array}$ & $\begin{array}{c}7 \\
10.8 \\
13 \\
20.0 \\
4 \\
7.4 \\
8 \\
14.8\end{array}$ & $\begin{array}{c}1 \\
1.5 \\
3 \\
4.6 \\
0 \\
0.0 \\
3 \\
5.6\end{array}$ & $\begin{array}{c}4 \\
6.2 \\
9 \\
13.9 \\
9 \\
16.7 \\
14 \\
25.9\end{array}$ \\
\hline $\begin{array}{l}89-97 \text { years } \\
(n=39)\end{array}$ & $\begin{array}{l}\text { Initial } \\
(\%) \\
\text { Subsequent } \\
(\%)\end{array}$ & $\begin{array}{c}4 \\
10.3 \\
2 \\
5.1\end{array}$ & $\begin{array}{c}4 \\
10.3 \\
3 \\
7.7\end{array}$ & $\begin{array}{c}11 \\
28.2 \\
4 \\
10.3\end{array}$ & $\begin{array}{c}6 \\
15.4 \\
4 \\
10.3\end{array}$ & $\begin{array}{c}0 \\
0.0 \\
2 \\
5.1\end{array}$ & $\begin{array}{c}0 \\
0.0 \\
0 \\
0.0\end{array}$ & $\begin{array}{c}7 \\
18.0 \\
13 \\
33.3\end{array}$ & $\begin{array}{c}1 \\
2.6 \\
5 \\
12.8\end{array}$ & $\begin{array}{c}6 \\
15.4 \\
6 \\
15.4\end{array}$ \\
\hline $\begin{array}{l}\text { All } \\
(n=158)\end{array}$ & $\begin{array}{l}\text { Initial } \\
(\%) \\
\text { Subsequent } \\
(\%)\end{array}$ & $\begin{array}{c}28 \\
17.7 \\
12 \\
7.6\end{array}$ & $\begin{array}{c}29 \\
18.4 \\
20 \\
12.7\end{array}$ & $\begin{array}{c}39 \\
24.7 \\
23 \\
14.6\end{array}$ & $\begin{array}{c}22 \\
13.9 \\
25 \\
15.8\end{array}$ & $\begin{array}{c}1 \\
0.6 \\
4 \\
2.5\end{array}$ & $\begin{array}{c}0 \\
0.0 \\
0 \\
0.0\end{array}$ & $\begin{array}{c}18 \\
11.4 \\
34 \\
21.5\end{array}$ & $\begin{array}{l}2 \\
1.3 \\
11 \\
7.0\end{array}$ & $\begin{array}{c}19 \\
12.0 \\
29 \\
18.4\end{array}$ \\
\hline
\end{tabular}

Grade: 0 , stippling only; 1 , drusen hard indistinct or questionable; 2 , hard distinct drusen, diameter $\leqslant 63 \mu \mathrm{m} ; 3$, drusen diameter $>63 \mu \mathrm{m}$ and $\leqslant 125 \mu \mathrm{m} ; 4$, drusen diameter $>125 \mu \mathrm{m}$ and $\leqslant 250 \mu ; 5$, drusen diameter $>250 \mu \mathrm{m} ; 6$, reticular drusen; $7 \& 8$, cannot grade (obscuring lesion and photograph quality amalgamated); NA, no drusen. Predominant drusen size assessment was made for all subfields combined.

drusen these were amalgamated with soft distinct drusen in the table (grades 2 and 3). Of note in Table IV is the shift up the rank-ordered scale between initial and subsequent examinations, with the prevalence of reticular drusen (grade 4) doubling to around $24 \%$ in the subsequent examination and a commensurate reduction in the prevalence of hard distinct drusen (Table IV, grade 1).

The area of retina involved by drusen is presented in two ways. In Table $\mathrm{V}$ the highest scoring subfield was used as the score for the eye as a whole. At the subsequent assessment, $20.3 \%$ of eyes had at least one subfield where drusen occupied over $50 \%$ of the subfield, which contrasted with the initial examination where the equivalent proportion of eyes was only $7.6 \%$. Nevertheless, at the subsequent examination 32 of the 99 eyes with drusen qualifying for area assessment had a maximum scoring subfield with drusen occupying $<3.1 \%$ of the area of that subfield (Table V, grade 3 or less). From the drusen area scores for the individual subfields, the percentage area occupied by definite drusen was calculated for the entire grid area. Fig. 2 provides a frequency distribution of these areas (on a geometric scale). 'Drusen absent' indicates gradable eyes where drusen were either absent or doubtful (hard indistinct, questionable or stippling only). As in Table V, there were drusen which qualified for area assessment in 115 eyes initially and 99 eyes subsequently. Where drusen where present the number of eyes with a total drusen area $<0.64 \%$ at initial assessment was 67 of $115(58.3 \%)$, whilst 13 of 115 eyes $(11.3 \%)$ had a drusen area of $10 \%$ or more, and 1 of 115 an area of over $40 \%$. At the subsequent examination equivalent figures were 37 of 99 eyes $(37.4 \%)$ with area $<0.64 \%, 32$ of $99(32.3 \%)$ with an area of $10 \%$

Table IV. Drusen type (per eye) for the three age groups at initial and subsequent assessments

\begin{tabular}{|c|c|c|c|c|c|c|c|}
\hline \multirow{2}{*}{$\begin{array}{l}\text { Age at subsequent } \\
\text { examination }\end{array}$} & & \multicolumn{6}{|c|}{ Grade of drusen type } \\
\hline & & 0 & 1 & $2 \& 3$ & 4 & $7 \& 8$ & NA \\
\hline $\begin{array}{l}84-85 \text { years } \\
(n=65)\end{array}$ & $\begin{array}{l}\text { Initial } \\
(\%) \\
\text { Subsequent } \\
(\%)\end{array}$ & $\begin{array}{c}15 \\
23.1 \\
7 \\
10.8\end{array}$ & $\begin{array}{c}17 \\
26.2 \\
5 \\
7.7\end{array}$ & $\begin{array}{c}20 \\
30.7 \\
27 \\
41.5\end{array}$ & $\begin{array}{c}8 \\
12.3 \\
14 \\
25.5\end{array}$ & $\begin{array}{c}1 \\
1.5 \\
3 \\
4.6\end{array}$ & $\begin{array}{c}4 \\
6.2 \\
9 \\
13.8\end{array}$ \\
\hline $\begin{array}{l}86-88 \text { years } \\
(n=54)\end{array}$ & $\begin{array}{l}\text { Initial } \\
\text { (\%) } \\
\text { Subsequent } \\
(\%)\end{array}$ & $\begin{array}{c}11 \\
20.4 \\
9 \\
16.7\end{array}$ & $\begin{array}{c}15 \\
27.8 \\
7 \\
13 . C\end{array}$ & $\begin{array}{c}15 \\
27.8 \\
10 \\
18.5\end{array}$ & $\begin{array}{c}4 \\
7.4 \\
11 \\
20.4\end{array}$ & $\begin{array}{c}0 \\
0.0 \\
3 \\
5.6\end{array}$ & $\begin{array}{c}9 \\
16.7 \\
14 \\
25.9\end{array}$ \\
\hline $\begin{array}{l}89-97 \text { years } \\
(n=39)\end{array}$ & $\begin{array}{l}\text { Initial } \\
\text { (\%) } \\
\text { Subsequent } \\
(\%)\end{array}$ & $\begin{array}{c}6 \\
15.4 \\
5 \\
12.8\end{array}$ & $\begin{array}{c}9 \\
23.1 \\
3 \\
7.7\end{array}$ & $\begin{array}{c}10 \\
25.6 \\
7 \\
18.0\end{array}$ & $\begin{array}{c}7 \\
18.0 \\
13 \\
33.3\end{array}$ & $\begin{array}{c}1 \\
2.6 \\
5 \\
12.8\end{array}$ & $\begin{array}{c}6 \\
15.4 \\
6 \\
15.4\end{array}$ \\
\hline $\begin{array}{l}\text { All } \\
(n=158)\end{array}$ & $\begin{array}{l}\text { Initial } \\
\text { (\%) } \\
\text { Subsequent } \\
\text { (\%) }\end{array}$ & $\begin{array}{c}32 \\
20.3 \\
21 \\
13.3\end{array}$ & $\begin{array}{c}41 \\
26.0 \\
15 \\
9.5\end{array}$ & $\begin{array}{c}45 \\
28.5 \\
43 \\
27.9\end{array}$ & $\begin{array}{c}19 \\
12.0 \\
38 \\
24.1\end{array}$ & $\begin{array}{l}2 \\
1.3 \\
11 \\
7.0\end{array}$ & $\begin{array}{c}19 \\
12.0 \\
29 \\
18.4\end{array}$ \\
\hline
\end{tabular}

Grade: 0 , hard indistinct or questionable drusen or stippling (drusen doubtful); 1 , hard distinct drusen; $2 \& 3$, soft distinct and soft indistinct drusen amalgamated; 4 , reticular drusen; $7 \& 8$, cannot grade (obscuring lesion and photograph quality amalgamated); NA, no drusen. Drusen type for the eye as a whole was taken from the 'highest'-scoring subfield (see text for rank-ordering of drusen type). 
Table V. Drusen area (per eye) for the three age groups at initial and subsequent assessments

\begin{tabular}{|c|c|c|c|c|c|c|c|c|c|c|c|}
\hline \multirow{2}{*}{$\begin{array}{l}\text { Age at subsequent } \\
\text { examination }\end{array}$} & & \multicolumn{10}{|c|}{ Grade of drusen area } \\
\hline & & 0 & 1 & 2 & 3 & 4 & 5 & 6 & 7 & 8 & NA \\
\hline $\begin{array}{l}84-85 \text { years } \\
(n=65)\end{array}$ & $\begin{array}{l}\text { Initial } \\
(\%) \\
\text { Subsequent } \\
(\%)\end{array}$ & $\begin{array}{c}1 \\
1.5 \\
1 \\
1.5\end{array}$ & $\begin{array}{c}10 \\
15.4 \\
5 \\
7.7\end{array}$ & $\begin{array}{c}6 \\
9.2 \\
6 \\
9.2\end{array}$ & $\begin{array}{c}8 \\
12.3 \\
2 \\
3.1\end{array}$ & $\begin{array}{c}9 \\
13.9 \\
2 \\
3.1\end{array}$ & $\begin{array}{c}2 \\
3.1 \\
9 \\
13.9\end{array}$ & $\begin{array}{c}3 \\
4.6 \\
5 \\
7.7\end{array}$ & $\begin{array}{c}3 \\
4.6 \\
5 \\
7.7\end{array}$ & $\begin{array}{c}7 \\
10.8 \\
11 \\
16.9\end{array}$ & $\begin{array}{c}16 \\
24.6 \\
19 \\
29.2\end{array}$ \\
\hline $\begin{array}{l}86-88 \text { years } \\
(n=54)\end{array}$ & $\begin{array}{l}\text { Initial } \\
(\%) \\
\text { Subsequent } \\
(\%)\end{array}$ & $\begin{array}{c}5 \\
9.3 \\
4 \\
7.4\end{array}$ & $\begin{array}{c}7 \\
13.0 \\
5 \\
9.3\end{array}$ & $\begin{array}{c}6 \\
11.1 \\
4 \\
7.4\end{array}$ & $\begin{array}{c}5 \\
9.3 \\
2 \\
3.7\end{array}$ & $\begin{array}{c}3 \\
5.6 \\
1 \\
1.9\end{array}$ & $\begin{array}{c}3 \\
5.6 \\
1 \\
1.9\end{array}$ & $\begin{array}{c}4 \\
7.4 \\
0 \\
0.0\end{array}$ & $\begin{array}{c}4 \\
7.4 \\
2 \\
3.7\end{array}$ & $\begin{array}{c}1 \\
1.9 \\
11 \\
20.4\end{array}$ & $\begin{array}{c}16 \\
29.6 \\
24 \\
44.4\end{array}$ \\
\hline $\begin{array}{l}89-97 \text { years } \\
(n=39)\end{array}$ & $\begin{array}{l}\text { Initial } \\
\text { (\%) } \\
\text { Subsequent } \\
(\%)\end{array}$ & $\begin{array}{c}2 \\
5.1 \\
0 \\
0.0\end{array}$ & $\begin{array}{c}1 \\
2.6 \\
1 \\
2.6\end{array}$ & $\begin{array}{c}6 \\
15.4 \\
2 \\
5.1\end{array}$ & $\begin{array}{c}4 \\
10.3 \\
0 \\
0.0\end{array}$ & $\begin{array}{c}2 \\
5.1 \\
2 \\
5.1\end{array}$ & $\begin{array}{c}8 \\
20.5 \\
3 \\
7.7\end{array}$ & $\begin{array}{c}0 \\
0.0 \\
3 \\
7.7\end{array}$ & $\begin{array}{c}1 \\
2.6 \\
2 \\
5.1\end{array}$ & $\begin{array}{c}4 \\
10.3 \\
10 \\
25.6\end{array}$ & $\begin{array}{c}11 \\
28.2 \\
16 \\
41.0\end{array}$ \\
\hline $\begin{array}{l}\text { All } \\
(n=158)\end{array}$ & $\begin{array}{l}\text { Initial } \\
\text { (\%) } \\
\text { Subsequent } \\
(\%)\end{array}$ & $\begin{array}{c}8 \\
5.1 \\
5 \\
3.1\end{array}$ & $\begin{array}{c}18 \\
11.4 \\
11 \\
6.7\end{array}$ & $\begin{array}{c}18 \\
11.4 \\
12 \\
7.6\end{array}$ & $\begin{array}{c}17 \\
10.8 \\
4 \\
2.5\end{array}$ & $\begin{array}{c}14 \\
8.9 \\
5 \\
3.1\end{array}$ & $\begin{array}{l}13 \\
8.2 \\
13 \\
8.2\end{array}$ & $\begin{array}{r}7 \\
4.4 \\
8 \\
5.1\end{array}$ & $\begin{array}{c}8 \\
5.1 \\
9 \\
5.7\end{array}$ & $\begin{array}{c}12 \\
7.6 \\
32 \\
20.3\end{array}$ & $\begin{array}{c}43 \\
27.2 \\
59 \\
37.3\end{array}$ \\
\hline
\end{tabular}

Grade: 0 , area covered by drusen $<\mathrm{C} 0$ (circle with diameter $63 \mu \mathrm{m}$ ); 1 , area $\geqslant \mathrm{C} 0$ and $<\mathrm{C} 1$ (diameter $12.5 \mu \mathrm{m}$ ); 2 , area $\geqslant \mathrm{C} 1$ and $<1.6 \%$ subfield area; 3 , area $\geqslant 1.6 \%$ and $<3.1 \%$ subfield area; 4 , area $\geqslant 3.1 \%$ and $<6.3 \%$ subfield area; 5 , area $\geqslant 6.3 \%$ and $<12.5 \%$ subfield area; 6 , area $\geqslant 12.5 \%$ and $<25 \%$ subfield area; 7 , area $\geqslant 25 \%$ and $<50 \%$ subfield area; 8 , area $\geqslant 50 \%$ subfield area; NA, no drusen or drusen ungradable (i.e. missing data) or drusen doubtful (hard indistinct or questionable or stippling only). Drusen area score for the eye as a whole was taken from the highest-scoring subfield.

or more, and 17 of $99(17.2 \%)$ with an area of $40 \%$ or more.

Data on drusen confluence appear in Table VI. Confluence was initially present in $37.3 \%$ of all eyes or $51.3 \%$ of eyes with drusen eligible for confluence assessment. On subsequent examination the equivalent figures were $41.8 \%$ and $66.7 \%$ respectively, the predominant form being reticular drusen.

Reticular drusen have been scored in Tables II, III, IV and VI. Tables II and VI (maximum drusen size and drusen confluence) are in complete agreement for reticular drusen as these tables each refer to the maximum score for the eye as a whole. Tables III and IV (predominant drusen size and predominant drusen type), however, are in minor disagreement because these scores are derived from the predominant rather than the 'highest rated' drusen. Furthermore, predominant drusen type was assessed on a subfield by subfield scoring, while predominant drusen size was scored for all subfields together. These apparent inconsistencies therefore remain compatible with accurate application of the grading rules.

\section{Pigmentary Disturbance}

The scores from the subfields showing the maximum amount of RPE degeneration per eye comprise Table VII, and demonstrate that the overall pre-

\section{Drusen \% Area Distribution}

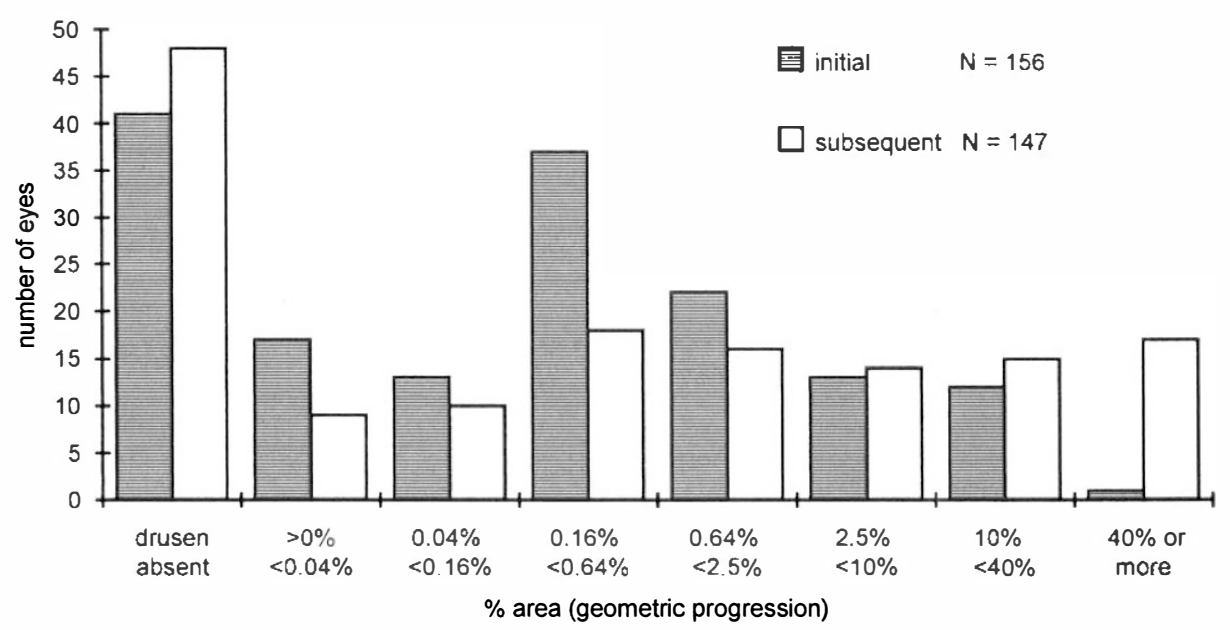

Fig. 2. Frequency distributions at initial and subsequent assessments of the percentage area covered by drusen for the entire grid area (within $3000 \mu \mathrm{m}$ of the fovea). Eyes ungradable for drusen were excluded and eyes where drusen were absent or doubtful are separately identified. At initial assessment there were 2 ungradable eyes, 41 eyes with drusen absent or doubtful and 115 eyes which qualified for drusen area assessment. At subsequent assessment there were 11 ungradable eyes, 48 eyes with drusen absent or doubtful and 99 eyes which qualified for drusen area assessment. 
Table VI. Drusen confluence (per eye) for the three age groups at initial and subsequent assessments

\begin{tabular}{|c|c|c|c|c|c|c|c|c|c|c|}
\hline \multirow{2}{*}{$\begin{array}{l}\text { Age at subsequent } \\
\text { examination }\end{array}$} & & \multicolumn{9}{|c|}{ Grade of drusen confluence } \\
\hline & & 0 & 1 & 2 & 3 & 4 & 5 & 6 & $7 \& 8$ & NA \\
\hline $\begin{array}{l}84-85 \text { years } \\
(n=65)\end{array}$ & $\begin{array}{l}\text { Initial } \\
(\%) \\
\text { Subsequent } \\
(\%)\end{array}$ & $\begin{array}{c}15 \\
23.1 \\
13 \\
20.0\end{array}$ & $\begin{array}{c}4 \\
6.2 \\
3 \\
4.6\end{array}$ & $\begin{array}{c}12 \\
18.5 \\
4 \\
6.2\end{array}$ & $\begin{array}{c}9 \\
13.9 \\
9 \\
13.9\end{array}$ & $\begin{array}{c}1 \\
1.5 \\
1 \\
1.5\end{array}$ & $\begin{array}{c}0 \\
0.0 \\
1 \\
1.5\end{array}$ & $\begin{array}{c}8 \\
12.3 \\
15 \\
23.1\end{array}$ & $\begin{array}{c}1 \\
1.5 \\
3 \\
4.6\end{array}$ & $\begin{array}{c}15 \\
23.1 \\
16 \\
24.6\end{array}$ \\
\hline $\begin{array}{l}86-88 \text { years } \\
(n=54)\end{array}$ & $\begin{array}{l}\text { Initial } \\
(\%) \\
\text { Subsequent } \\
(\%)\end{array}$ & $\begin{array}{c}17 \\
31.5 \\
9 \\
16.7\end{array}$ & $\begin{array}{c}5 \\
9.3 \\
3 \\
5.6\end{array}$ & $\begin{array}{c}6 \\
11.1 \\
4 \\
7.4\end{array}$ & $\begin{array}{c}5 \\
9.3 \\
2 \\
3.7\end{array}$ & $\begin{array}{c}1 \\
1.9 \\
0 \\
0.0\end{array}$ & $\begin{array}{c}0 \\
0.0 \\
0 \\
0.0\end{array}$ & $\begin{array}{c}4 \\
7.4 \\
12 \\
22.2\end{array}$ & $\begin{array}{c}0 \\
0.0 \\
3 \\
5.6\end{array}$ & $\begin{array}{c}16 \\
29.6 \\
21 \\
38.9\end{array}$ \\
\hline 89-97 years & $\begin{array}{l}\text { Initial } \\
\text { (\%) } \\
\text { Subsequent } \\
(\%)\end{array}$ & $\begin{array}{c}8 \\
20.5 \\
4 \\
10.3\end{array}$ & $\begin{array}{c}7 \\
18.0 \\
1 \\
2.6\end{array}$ & $\begin{array}{c}2 \\
5.1 \\
2 \\
5.1\end{array}$ & $\begin{array}{c}4 \\
10.3 \\
3 \\
7.7\end{array}$ & $\begin{array}{c}0 \\
0.0 \\
0 \\
0.0\end{array}$ & $\begin{array}{c}0 \\
0.0 \\
0 \\
0.0\end{array}$ & $\begin{array}{c}7 \\
18.0 \\
13 \\
33.3\end{array}$ & $\begin{array}{c}1 \\
2.6 \\
5 \\
12.8\end{array}$ & $\begin{array}{c}10 \\
25.6 \\
11 \\
28.2\end{array}$ \\
\hline $\begin{array}{l}\text { All } \\
(n=158)\end{array}$ & $\begin{array}{l}\text { Initial } \\
\text { (\%) } \\
\text { Subsequent } \\
(\%)\end{array}$ & $\begin{array}{c}40 \\
25.3 \\
26 \\
16.5\end{array}$ & $\begin{array}{c}16 \\
10.1 \\
7 \\
4.4\end{array}$ & $\begin{array}{c}20 \\
12.7 \\
10 \\
6.3\end{array}$ & $\begin{array}{c}18 \\
11.4 \\
14 \\
8.9\end{array}$ & $\begin{array}{c}2 \\
1.3 \\
1 \\
0.6\end{array}$ & $\begin{array}{c}0 \\
0.0 \\
1 \\
0.6\end{array}$ & $\begin{array}{c}0 \\
12.0 \\
40 \\
25.3\end{array}$ & $\begin{array}{l}19 \\
1.3 \\
11 \\
7.0\end{array}$ & $\begin{array}{c}41 \\
25.9 \\
48 \\
30.4\end{array}$ \\
\hline
\end{tabular}

Grade: 0 , no confluence; 1 , questionable; 2 , confluence $<250 \mu \mathrm{m}$ diameter; 3 , confluence $\geqslant 250 \mu \mathrm{m}$ and $<500 \mu \mathrm{m}$ diameter; 4 , confluence $\geqslant 500 \mu \mathrm{m}$ and $<1000 \mu \mathrm{m}$ diameter; 5 , confluence $\geqslant 1000 \mu \mathrm{m}$, reticular drusen; $7 \& 8$, cannot grade (obscuring lesion and photograph quality amalgamated); NA, no drusen or drusen doubtful (hard indistinct or questionable or stippling only). Confluence assessment was made for all subfields combined.

valence of RPE degeneration (grades 2-4) at the second examination was $72.8 \%$ - much higher than the initial prevalence of $51.3 \%$. The number of eyes with a maximum subfield score of $>50 \%$ of that subfield area (grade 4) almost doubled between initial and subsequent examinations.

Increased pigment scores are shown in Table VIII. Definite pigment clumping due to AMD (grades 2 and 3) was a feature of $22.2 \%$ of eyes at first examination. In 28 of these 35 eyes $(80 \%)$, this pigmentation involved the central and inner fields, i.e. within $1500 \mu \mathrm{m}$ of the fovea. At the subsequent examination definite pigment clumping was observed in $16.5 \%$ of eyes.

\section{Exudative Disease}

Exudative disease was uncommon in this populationbased group (Table IX). Definite subretinal scars increased from 3 to 5 between initial and subsequent examinations (all were grade 4, middle part of Table IX). Excluding subretinal haemorrhage from other causes, only 1 eye had a definite subretinal haemorrhage, this being observed at the subsequent examination (grade 2, upper part of Table IX). Exudative lesions tended to involve the central and inner subfields when present.

\section{Geographic Atrophy}

Geographic atrophy was also uncommon, and affected 3 eyes initially and 5 at subsequent examination (grades 2-5, lower part of Table IX). The central and inner subfields were involved in all cases.

\section{Visual Acuity}

Snellen visual acuity distributions at the two points in time appear in Fig. 3. From these distributions it is

Table VII. RPE degeneration (per eye) for the three age groups at initial and subsequent assessments

\begin{tabular}{|c|c|c|c|c|c|c|c|}
\hline \multirow{2}{*}{$\begin{array}{l}\text { Age at subsequent } \\
\text { examination }\end{array}$} & & \multicolumn{6}{|c|}{ Grade of RPE degeneration } \\
\hline & & 0 & 1 & 2 & 3 & 4 & $7 \& 8$ \\
\hline $\begin{array}{l}84-85 \text { years } \\
(n=65)\end{array}$ & $\begin{array}{l}\text { Initial } \\
\text { (\%) } \\
\text { Subsequent } \\
(\%)\end{array}$ & $\begin{array}{c}35 \\
49.2 \\
13 \\
20.0\end{array}$ & $\begin{array}{c}1 \\
1.5 \\
5 \\
7.7\end{array}$ & $\begin{array}{c}12 \\
18.5 \\
7 \\
10.8\end{array}$ & $\begin{array}{c}2 \\
3.1 \\
0 \\
0.0\end{array}$ & $\begin{array}{c}18 \\
27.7 \\
39 \\
60.0\end{array}$ & $\begin{array}{c}0 \\
0.0 \\
1 \\
1.5\end{array}$ \\
\hline $\begin{array}{l}86-88 \text { years } \\
(n=54)\end{array}$ & $\begin{array}{l}\text { Initial } \\
\text { (\%) } \\
\text { Subsequent } \\
(\%)\end{array}$ & $\begin{array}{c}22 \\
40.7 \\
11 \\
20.4\end{array}$ & $\begin{array}{c}4 \\
7.4 \\
3 \\
5.6\end{array}$ & $\begin{array}{c}6 \\
11.1 \\
3 \\
5.6\end{array}$ & $\begin{array}{c}2 \\
3.7 \\
4 \\
7.4\end{array}$ & $\begin{array}{c}20 \\
37.0 \\
33 \\
61.1\end{array}$ & $\begin{array}{c}0 \\
0.0 \\
0 \\
0.0\end{array}$ \\
\hline $\begin{array}{l}89-97 \text { years } \\
(n=39)\end{array}$ & $\begin{array}{l}\text { Initial } \\
\text { (\%) } \\
\text { Subsequent } \\
(\%)\end{array}$ & $\begin{array}{c}14 \\
35.9 \\
8 \\
20.5\end{array}$ & $\begin{array}{c}4 \\
10.3 \\
2 \\
5.1\end{array}$ & $\begin{array}{c}4 \\
10.3 \\
3 \\
7.7\end{array}$ & $\begin{array}{c}1 \\
2.6 \\
1 \\
2.6\end{array}$ & $\begin{array}{c}16 \\
41.0 \\
25 \\
64.1\end{array}$ & $\begin{array}{c}0 \\
0.0 \\
0 \\
0.0\end{array}$ \\
\hline $\begin{array}{l}\text { All } \\
(n=158)\end{array}$ & $\begin{array}{l}\text { Initial } \\
(\%) \\
\text { Subsequent } \\
(\%)\end{array}$ & $\begin{array}{c}71 \\
43.0 \\
32 \\
20.3\end{array}$ & $\begin{array}{c}9 \\
5.7 \\
10 \\
6.3\end{array}$ & $\begin{array}{c}22 \\
13.9 \\
13 \\
8.2\end{array}$ & $\begin{array}{c}5 \\
3.2 \\
5 \\
3.2\end{array}$ & $\begin{array}{c}54 \\
34.2 \\
97 \\
61.4\end{array}$ & $\begin{array}{c}0 \\
0.0 \\
1 \\
0.6\end{array}$ \\
\hline
\end{tabular}

Grade: 0, no RPE degeneration; 1, questionable RPE degeneration; 2, RPE degeneration in $<25 \%$ of subfield; 3, RPE degeneration in $<50 \%$ of subfield; 4 , RPE degeneration in $\geqslant 50 \%$ of subfield; $7 \& 8$, cannot grade (obscuring lesion and photograph quality amalgamated). 
Table VIII. Increased pigmentation for all ages in three concentric retinal zones

\begin{tabular}{|c|c|c|c|c|c|c|c|}
\hline \multirow{2}{*}{\multicolumn{3}{|c|}{. }} & \multicolumn{5}{|c|}{ Increased pigmentation } \\
\hline & & & 0 & 1 & 2 & 3 & $6,7,8$ \\
\hline $\begin{array}{l}\text { All subfields } \\
(n=158)\end{array}$ & $\begin{array}{l}\text { Initial } \\
\text { Subsequent }\end{array}$ & $\begin{array}{l}(n) \\
(\%) \\
(n) \\
(\%)\end{array}$ & $\begin{array}{c}116 \\
73.4 \\
126 \\
79.8\end{array}$ & $\begin{array}{c}6 \\
3.8 \\
4 \\
2.5\end{array}$ & $\begin{array}{c}17 \\
10.8 \\
11 \\
6.7\end{array}$ & $\begin{array}{c}18 \\
11.4 \\
15 \\
9.5\end{array}$ & $\begin{array}{c}1 \\
0.6 \\
2 \\
1.3\end{array}$ \\
\hline $\begin{array}{l}\text { Central \& inner } \\
(n=158)\end{array}$ & $\begin{array}{l}\text { Initial } \\
\text { Subsequent }\end{array}$ & $\begin{array}{l}(n) \\
(\%) \\
(n) \\
(\%)\end{array}$ & $\begin{array}{c}124 \\
78.5 \\
130 \\
82.8\end{array}$ & $\begin{array}{c}5 \\
3.2 \\
3 \\
1.9\end{array}$ & $\begin{array}{l}14 \\
8.9 \\
10 \\
6.3\end{array}$ & $\begin{array}{l}14 \\
8.9 \\
12 \\
7.6\end{array}$ & $\begin{array}{c}1 \\
0.6 \\
3 \\
1.9\end{array}$ \\
\hline $\begin{array}{l}\text { Central only } \\
(n=153)^{\mathrm{a}}\end{array}$ & $\begin{array}{l}\text { Initial } \\
\text { Subsequent }\end{array}$ & $\begin{array}{l}(n) \\
(\%) \\
(n) \\
(\%)\end{array}$ & $\begin{array}{c}130 \\
85.0 \\
139 \\
90.8\end{array}$ & $\begin{array}{c}5 \\
3.3 \\
3 \\
2.0\end{array}$ & $\begin{array}{c}14 \\
9.1 \\
5 \\
3.3\end{array}$ & $\begin{array}{c}4 \\
2.6 \\
6 \\
3.9\end{array}$ & $\begin{array}{c}0 \\
0.0 \\
0 \\
0.0\end{array}$ \\
\hline
\end{tabular}

Grade: 0 , no pigment; 1 , questionable pigment; 2 , a rea of pigment $<250 \mu \mathrm{m} ; 3$, a rea of pigment $\geqslant 250 \mu \mathrm{m} ; 6,7,8$, cannot grade (pigment, other obscuring lesion and photograph quality amalgamated). For the 'all subfields' and 'central \& inner' zones the highest score for any subfield was used to provide the grade for each zone on a 'by eye' basis.

${ }^{a}$ Missing data on central fields for 5 eyes for both initial and subsequent assessments.

clear that the acuity was generally better at the initial assessments. The prevalence of eyes with normal acuity $(6 / 6$ or better) was initially $13.3 \%$, whilst only $5.7 \%$ of eyes had this acuity subsequently. Also, $70.9 \%$ of eyes had an acuity of $6 / 12$ or better (required for driving in the UK) at initial assessment, in contrast to approximately $50.6 \%$ of eyes with this acuity subsequently. The prevalence of blindness (6/ 60 or worse) was double at subsequent assessment ( $10.1 \%$ initially vs $20.9 \%$ subsequently).

\section{$A M D$ and Visual Acuity}

Table X summarises the prevalence of specific AMD features in combination with visual acuity thresholds in this elderly population at the two time points. Not surprisingly, no eyes with either exudative AMD (subretinal haemorrhage, fibrin or scar) or geographic atrophy, had a visual acuity of $6 / 12$ or better. It is also of note that whilst RPE degeneration was present at subsequent assessment in roughly $85 \%$ of legally blind eyes (acuity $6 / 60$ or worse), it was similarly present in approximately $85 \%$ of eyes with only mild visual impairment (acuity 6/9-6/12).

In Tables XI and XII, AMD features were grouped to produce summary statements of AMD prevalence at various' levels of visual acuity. Table XI refers to all eyes and Table XII to the better eye of each individual. The term 'No AMD' encompassed absent or questionable grades, and for drusen this included indistinct hard drusen and stippling. Any AMD was defined by a 'definitely present' score for any feature. This was subdivided into minor AMD and major AMD. Minor AMD encompassed any of the following features: non-confluent drusen or drusen with confluence $<500 \mu \mathrm{m}$, definite drusen which were not reticular, and a total drusen area covering $<10 \%$ of all subfields combined; RPE degeneration covering $<25 \%$ of all subfields combined; and increased

Table IX. Exudative AMD features (subretinal scar/fibrin deposit and subretinal haemorrhage) and geographic atrophy for all ages and all subfields combined

\begin{tabular}{|c|c|c|c|c|c|c|c|}
\hline \multirow[t]{2}{*}{ Feature } & & & \multicolumn{5}{|c|}{ Grade } \\
\hline & & & 0 & 1 & 2 & & $7 \& 8$ \\
\hline Subretinal haemorrhage & $\begin{array}{l}\text { Initial } \\
\text { Subsequent }\end{array}$ & $\begin{array}{l}(n) \\
(\%) \\
(n) \\
(\%)\end{array}$ & $\begin{array}{c}157 \\
99.4 \\
155 \\
98.1\end{array}$ & $\begin{array}{c}0 \\
0.0 \\
1 \\
0.6\end{array}$ & $\begin{array}{c}1^{\mathrm{a}} \\
0.6 \\
1 \\
0.6\end{array}$ & & $\begin{array}{c}0 \\
0.0 \\
1 \\
0.6\end{array}$ \\
\hline & & & 0 & 1 & 2,3 & 4 & $7 \& 8$ \\
\hline $\begin{array}{l}\text { Subretinal scar } \\
(n=158)\end{array}$ & $\begin{array}{l}\text { Initial } \\
\text { Subsequent }\end{array}$ & $\begin{array}{l}(n) \\
(\%) \\
(n) \\
(\%)\end{array}$ & $\begin{array}{c}154 \\
97.5 \\
151 \\
95.6 \\
\end{array}$ & $\begin{array}{c}1 \\
0.6 \\
2 \\
1.3 \\
\end{array}$ & $\begin{array}{c}0 \\
0.0 \\
0 \\
0.0 \\
\end{array}$ & $\begin{array}{c}3 \\
1.9 \\
5 \\
3.2 \\
\end{array}$ & $\begin{array}{c}0 \\
0.0 \\
0 \\
0.0 \\
\end{array}$ \\
\hline & & & 0 & 1 & $2,3,4$ & 5 & $7 \& 8$ \\
\hline $\begin{array}{l}\text { Geographic atrophy } \\
(n=158)\end{array}$ & $\begin{array}{l}\text { Initial } \\
\text { Subsequent }\end{array}$ & $\begin{array}{l}(n) \\
(\%) \\
(n) \\
(\%)\end{array}$ & $\begin{array}{c}154 \\
97.5 \\
151 \\
95.6\end{array}$ & $\begin{array}{c}1 \\
0.6 \\
1 \\
0.6\end{array}$ & $\begin{array}{c}0 \\
0.0 \\
0 \\
0.0\end{array}$ & $\begin{array}{c}3 \\
1.9 \\
5 \\
3.2\end{array}$ & $\begin{array}{c}0 \\
0.0 \\
1 \\
0.6\end{array}$ \\
\hline
\end{tabular}

Grade: 0 , feature absent; 1 , feature questionably present; 2 (upper part), subretinal haemorrhage present; 2,3 (middle part, amalgamated), subretinal scar/fibrin present $<50 \%$ subfield; 4 (middle part), scar/fibrin $\geqslant 50 \%$ subfield; $2,3,4$ (lower part, amalgamated), geographic atrophy present $<50 \%$ of subfield; 5 (lower part), geographic atrophy present $\geqslant 50 \%$ subfield; $7 \& 8$, cannot grade (obscuring lesion and photograph quality amalgamated).

'This subretinal haemorrhage was not due to AMD. The highest score for any subfield was used to provide the grade on a 'by eye' basis. 


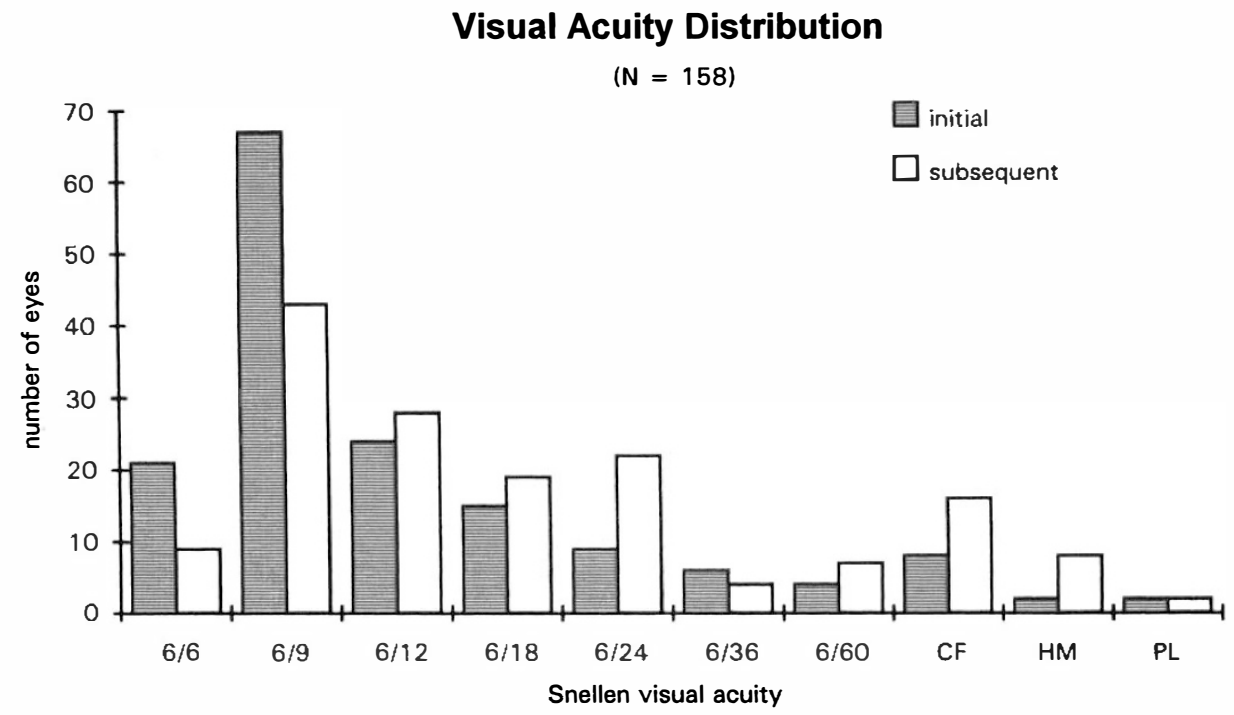

Fig. 3. Frequency distributions of Snellen visual acuity at initial and subsequent assessments

pigmentation. Major AMD was defined by the presence of reticular drusen, drusen with confluence $\geqslant 500 \mu \mathrm{m}$, or drusen together occupying $\geqslant 10 \%$ total subfield area; RPE degeneration involving $\geqslant 25 \%$ of combined subfields; exudative disease (subretinal scar/fibrin or subretinal haemorrhage); and/or geographic atrophy. Denominators in these tables refer to the number of eyes with the specified level of vision for the column. From Table XI, it is apparent that at initial assessment 30 eyes $(19.0 \%)$ had moderate visual impairment (6/18-6/36), of which roughly half had minor AMD and one third major
AMD. At the subsequent assessment, although the number of eyes with this level of acuity had risen to $45(28.5 \%)$, the proportions of eyes with both minor AMD and major AMD remained approximately the same. This contrasts with the situation in the eyes with severe visual impairment. Initially 19 eyes $(12.0 \%)$ were blind (6/60 or worse), of which 10 of $19(52.6 \%)$ showed minor AMD and 7 of $19(36.8 \%)$ showed major AMD. At follow-up, only 3 of 33 $(9.1 \%)$ blind eyes showed minor AMD changes, whilst 27 of $33(81.8 \%)$ eyes showed major AMD features. It is also of note, however, that $42(50.6 \%)$

Table X. Prevalence of AMD features in relation to visual acuity

\begin{tabular}{|c|c|c|c|c|c|c|c|}
\hline \multirow{2}{*}{ Feature $(n=158)$} & & & \multicolumn{5}{|c|}{ Visual acuity } \\
\hline & & & $6 / 6$ or better & $6 / 9-6 / 12$ & $6 / 18-6 / 36$ & $6 / 60$ or worse & All visions \\
\hline Any drusen & $\begin{array}{l}\text { Initial } \\
\text { Subsequent }\end{array}$ & $\begin{array}{l}(n) \\
(\%) \\
(n) \\
(\%)\end{array}$ & $\begin{array}{c}17 / 21 \\
81.0 \% \\
7 / 9 \\
77.8 \%\end{array}$ & $\begin{array}{c}81 / 88 \\
92.0 \% \\
54 / 71 \\
76.1 \%\end{array}$ & $\begin{array}{c}6 / 30 \\
20.0 \% \\
19 / 45 \\
42.2 \%\end{array}$ & $\begin{array}{c}15 / 19 \\
78.9 \% \\
22 / 33 \\
6.7 \%\end{array}$ & $\begin{array}{c}116 / 158 \\
73.4 \% \\
102 / 158 \\
64.6 \%\end{array}$ \\
\hline Drusen confluence & $\begin{array}{l}\text { Initial } \\
\text { Subsequent }\end{array}$ & $\begin{array}{l}(n) \\
(\%) \\
(n) \\
(\%)\end{array}$ & $\begin{array}{c}6 / 21 \\
28.6 \% \\
3 / 9 \\
33.3 \%\end{array}$ & $\begin{array}{c}42 / 88 \\
47.7 \% \\
32 / 71 \\
45.1 \%\end{array}$ & $\begin{array}{c}4 / 30 \\
13.3 \% \\
12 / 45 \\
26.7 \%\end{array}$ & $\begin{array}{c}8 / 19 \\
42.1 \% \\
19 / 33 \\
57.6 \%\end{array}$ & $\begin{array}{c}60 / 158 \\
38.0 \% \\
66 / 158 \\
41.8 \%\end{array}$ \\
\hline RPE degeneration & $\begin{array}{l}\text { Initial } \\
\text { Subsequent }\end{array}$ & $\begin{array}{l}(n) \\
(\%) \\
(n) \\
(\%)\end{array}$ & $\begin{array}{c}10 / 21 \\
47.6 \% \\
4 / 9 \\
44.4 \%\end{array}$ & $\begin{array}{c}51 / 88 \\
58.0 \% \\
61 / 71 \\
85.9 \%\end{array}$ & $\begin{array}{c}8 / 30 \\
26.7 \% \\
23 / 45 \\
51.5 \%\end{array}$ & $\begin{array}{c}19 / 19 \\
68.4 \% \\
28 / 33 \\
84.8 \%\end{array}$ & $\begin{array}{c}82 / 158 \\
51.9 \% \\
116 / 158 \\
73.4 \%\end{array}$ \\
\hline Increased pigment & $\begin{array}{l}\text { Initial } \\
\text { Subsequent }\end{array}$ & $\begin{array}{l}(n) \\
(\%) \\
(n) \\
(\%)\end{array}$ & $\begin{array}{c}5 / 21 \\
23.8 \% \\
2 / 9 \\
22.2 \%\end{array}$ & $\begin{array}{c}19 / 88 \\
21.6 \% \\
9 / 71 \\
12.7 \%\end{array}$ & $\begin{array}{c}1 / 30 \\
3.3 \% \\
2 / 45 \\
4.4 \%\end{array}$ & $\begin{array}{c}11 / 19 \\
57.9 \% \\
13 / 33 \\
39.4 \%\end{array}$ & $\begin{array}{c}36 / 158 \\
22.8 \% \\
26 / 158 \\
16.5 \%\end{array}$ \\
\hline Exudative $\mathrm{AMD}^{\mathrm{a}}$ & $\begin{array}{l}\text { Initial } \\
\text { Subsequent }\end{array}$ & $\begin{array}{l}(n) \\
(\%) \\
(n) \\
(\%)\end{array}$ & $\begin{array}{c}0 / 21 \\
0.0 \% \\
0 / 9 \\
0.0 \%\end{array}$ & $\begin{array}{c}0 / 88 \\
0.0 \% \\
0 / 71 \\
0.0 \%\end{array}$ & $\begin{array}{c}0 / 30 \\
0.0 \% \\
1 / 45 \\
2.2 \%\end{array}$ & $\begin{array}{r}3 / 19 \\
15.8 \% \\
5 / 33 \\
12.5 \%\end{array}$ & $\begin{array}{c}3 / 158 \\
1.9 \% \\
6 / 158 \\
3.8 \%\end{array}$ \\
\hline Geographic atrophy & $\begin{array}{l}\text { Initial } \\
\text { Subsequent }\end{array}$ & $\begin{array}{l}(n) \\
(\%) \\
(n) \\
(\%)\end{array}$ & $\begin{array}{c}0 / 21 \\
0.0 \% \\
0 / 9 \\
0.0 \%\end{array}$ & $\begin{array}{c}0 / 88 \\
0.0 \% \\
0 / 71 \\
0.0 \%\end{array}$ & $\begin{array}{c}1 / 30 \\
3.3 \% \\
0 / 45 \\
0.0 \%\end{array}$ & $\begin{array}{c}2 / 19 \\
10.5 \% \\
5 / 33 \\
15.2 \%\end{array}$ & $\begin{array}{l}3 / 158 \\
1.9 \% \\
5 / 158 \\
3.2 \%\end{array}$ \\
\hline
\end{tabular}

Items in the table refer to the prevalence of the feature in eyes with the stated level of vision.

"Subretinal scar/fibrin and/or haemorrhage (one eye with subretinal haemorrhage not due to AMD was excluded). 
Table XI. Summary of the prevalence of AMD subtypes at various levels of visual acuity: analysis by eye

\begin{tabular}{|c|c|c|c|c|c|c|c|}
\hline \multirow[b]{2}{*}{ AMD type $(n=158)$} & & & \multicolumn{5}{|c|}{ Visual acuity } \\
\hline & & & $6 / 6$ or better & $6 / 9-6 / 12$ & $6 / 18-6 / 36$ & $6 / 60$ or worse & All visions \\
\hline No AMD & $\begin{array}{l}\text { Initial } \\
\text { Subsequent }\end{array}$ & $\begin{array}{l}(n) \\
(\%) \\
(n) \\
(\%)\end{array}$ & $\begin{array}{c}2 / 21 \\
9.5 \% \\
3 / 9 \\
33.3 \%\end{array}$ & $\begin{array}{c}16 / 88 \\
18.2 \% \\
7 / 71 \\
9.9 \%\end{array}$ & $\begin{array}{c}7 / 30 \\
23.3 \% \\
10 / 45 \\
22.2 \%\end{array}$ & $\begin{array}{c}2 / 19 \\
10.5 \% \\
3 / 33 \\
9.1 \%\end{array}$ & $\begin{array}{c}27 / 158 \\
17.1 \% \\
23 / 158 \\
14.6 \%\end{array}$ \\
\hline Minor AMD & $\begin{array}{l}\text { Initial } \\
\text { Subsequent }\end{array}$ & $\begin{array}{l}(n) \\
(\%) \\
(n) \\
(\%)\end{array}$ & $\begin{array}{c}12 / 21 \\
57.1 \% \\
3 / 9 \\
33.3 \%\end{array}$ & $\begin{array}{c}55 / 88 \\
62.5 \% \\
25 / 71 \\
35.2 \%\end{array}$ & $\begin{array}{c}14 / 30 \\
46.7 \% \\
21 / 45 \\
46.7 \%\end{array}$ & $\begin{array}{c}10 / 19 \\
52.6 \% \\
3 / 33 \\
9.1 \%\end{array}$ & $\begin{array}{c}91 / 158 \\
57.6 \% \\
52 / 158 \\
32.9 \%\end{array}$ \\
\hline Major AMD & $\begin{array}{l}\text { Initial } \\
\text { Subsequent }\end{array}$ & $\begin{array}{l}(n) \\
(\%) \\
(n) \\
(\%)\end{array}$ & $\begin{array}{c}7 / 21 \\
33.3 \% \\
3 / 9 \\
33.3 \%\end{array}$ & $\begin{array}{c}17 / 88 \\
19.8 \% \\
39 / 71 \\
54.9 \%\end{array}$ & $\begin{array}{c}9 / 30 \\
30.0 \% \\
14 / 45 \\
31.1 \%\end{array}$ & $\begin{array}{c}7 / 19 \\
36.8 \% \\
27 / 33 \\
81.8 \%\end{array}$ & $\begin{array}{c}40 / 158 \\
25.3 \% \\
83 / 158 \\
52.5 \%\end{array}$ \\
\hline Any AMD & $\begin{array}{l}\text { Initial } \\
\text { Subsequent }\end{array}$ & $\begin{array}{l}(n) \\
(\%) \\
(n) \\
(\%)\end{array}$ & $\begin{array}{c}19 / 21 \\
90.5 \% \\
6 / 9 \\
66.7 \%\end{array}$ & $\begin{array}{c}72 / 88 \\
81.8 \% \\
64 / 71 \\
90.1 \%\end{array}$ & $\begin{array}{c}23 / 30 \\
76.7 \% \\
35 / 45 \\
77.8 \%\end{array}$ & $\begin{array}{c}17 / 19 \\
89.5 \% \\
30 / 33 \\
90.9 \%\end{array}$ & $\begin{array}{c}131 / 158 \\
82.9 \% \\
135 / 158 \\
85.4 \%\end{array}$ \\
\hline
\end{tabular}

No $A M D$, AMD features either absent or questionably present (for drusen this included stippling and indistinct hard drusen).

Any $A M D$, any feature scored as being definitely present; this was subdivided into:

Minor $A M D$, definite drusen which were not reticular; with confluence $<50 \mu \mathrm{m}$ and overall percentage drusen area $<10 \%$ (all subfields combined); RPE degeneration with overall area involving $<25 \%$ of all subfields combined; and any increased pigmentation.

Major $A M D$, exudative disease in any subfield (subretinal scar/fibrin or subretinal haemorrhage); geographic atrophy in any subfield; RPE degeneration involving $25 \%$ or more of combined subfields; reticular drusen or drusen with confluence over $500 \mu \mathrm{m}$ and/or overall percentage drusen area $>10 \%$.

Items in the table refer to the prevalence of the subtypes of AMD in eyes stratified according to the stated level of vision.

of the 83 eyes with major AMD at the subsequent assessment maintained vision of 6/12 or better despite these changes. These results show that the development of major AMD is compatible with only mild visual impairment, but that the risk of severe visual impairment is increased.

Table XII illustrates AMD subtypes at various levels of vision in the better eye of each subject on the two occasions. Despite referring to the subjects' better eye, the prevalence of any AMD remains at or above $75 \%$ for all categories of vision on each occasion. The expectation that worse vision is associated with higher rates of major AMD is met on both occasions; furthermore, the overall rates shift from minor towards major AMD between initial and subsequent assessments.

Table XII. Summary of the prevalence of AMD subtypes at various levels of visual acuity: analysis by patient using the better eye

\begin{tabular}{|c|c|c|c|c|c|c|c|}
\hline \multirow[b]{2}{*}{ AMD type $(n=82)$} & & & \multicolumn{5}{|c|}{ Visual acuity } \\
\hline & & & $6 / 6$ or better & $6 / 9-6 / 12$ & $6 / 18-6 / 36$ & $6 / 60$ or worse & All visions \\
\hline No AMD & $\begin{array}{l}\text { Initial } \\
\text { Subsequent }\end{array}$ & $\begin{array}{l}(n) \\
(\%) \\
(n) \\
(\%)\end{array}$ & $\begin{array}{c}2 / 18 \\
11.1 \% \\
2 / 8 \\
25.0 \%\end{array}$ & $\begin{array}{r}9 / 49 \\
18.4 \% \\
5 / 41 \\
12.2 \%\end{array}$ & $\begin{array}{c}2 / 11 \\
18.2 \% \\
5 / 21 \\
23.8 \%\end{array}$ & $\begin{array}{c}0 / 4 \\
0.0 \% \\
2 / 12 \\
16.7 \%\end{array}$ & $\begin{array}{c}13 / 82 \\
15.9 \% \\
14 / 82 \\
17.1 \%\end{array}$ \\
\hline Minor AMD & $\begin{array}{l}\text { Initial } \\
\text { Subsequent }\end{array}$ & $\begin{array}{l}(n) \\
(\%) \\
(n) \\
(\%)\end{array}$ & $\begin{array}{c}10 / 18 \\
55.6 \% \\
3 / 8 \\
37.5 \%\end{array}$ & $\begin{array}{c}31 / 49 \\
63.4 \% \\
14 / 41 \\
34.1 \%\end{array}$ & $\begin{array}{c}4 / 11 \\
36.4 \% \\
9 / 21 \\
42.9 \%\end{array}$ & $\begin{array}{c}2 / 4 \\
50.0 \% \\
0 / 12 \\
0.0 \%\end{array}$ & $\begin{array}{c}47 / 82 \\
57.3 \% \\
26 / 82 \\
31.7 \%\end{array}$ \\
\hline Major AMD & $\begin{array}{l}\text { Initial } \\
\text { Subsequent }\end{array}$ & $\begin{array}{l}(n) \\
(\%) \\
(n) \\
(\%)\end{array}$ & $\begin{array}{c}6 / 18 \\
33.3 \% \\
3 / 8 \\
37.5 \%\end{array}$ & $\begin{array}{c}9 / 49 \\
18.4 \% \\
22 / 41 \\
53.7 \%\end{array}$ & $\begin{array}{c}5 / 11 \\
45.5 \% \\
7 / 11 \\
33.3 \%\end{array}$ & $\begin{array}{c}2 / 4 \\
50.0 \% \\
10 / 12 \\
83.3 \%\end{array}$ & $\begin{array}{c}22 / 82 \\
26.8 \% \\
42 / 82 \\
51.2 \%\end{array}$ \\
\hline Any AMD & $\begin{array}{l}\text { Initial } \\
\text { Subsequent }\end{array}$ & $\begin{array}{l}(n) \\
(\%) \\
(n) \\
(\%)\end{array}$ & $\begin{array}{c}16 / 18 \\
88.9 \% \\
6 / 8 \\
75.0 \%\end{array}$ & $\begin{array}{c}40 / 49 \\
81.6 \% \\
36 / 41 \\
87.8 \%\end{array}$ & $\begin{array}{c}9 / 11 \\
81.8 \% \\
16 / 21 \\
76.2 \%\end{array}$ & $\begin{array}{c}4 / 4 \\
100 \% \\
10 / 12 \\
83.3 \%\end{array}$ & $\begin{array}{c}69 / 82 \\
84.1 \% \\
68 / 82 \\
82.9 \%\end{array}$ \\
\hline
\end{tabular}

No $A M D$, AMD features either absent or questionably present (for drusen this included stippling and indistinct hard drusen). Any $A M D$, any feature scored as being definitely present; this was subdivided into:

Minor AMD, definite drusen which were not reticular; with confluence $<500 \mu \mathrm{m}$ and overall percentage drusen area $<10 \%$ (all subfields combined); RPE degeneration with overall area involving $<25 \%$ of all subfields combined; and any increased pigmentation.

Major $A M D$, exudative disease in any subfield (subretinal scar/fibrin or subretinal haemorrhage); geographic atrophy in any subfield; RPE degeneration involving $25 \%$ or more of combined subfields; reticular drusen or drusen with confluence over $500 \mu \mathrm{m}$ and/or overal percentage drusen area $>10 \%$

Items in the table refer to the prevalence of the subtypes of AMD in the patient's better eye. Patients were stratified according to the stated level of vision. 


\section{DISCUSSION}

The most distinctive feature of this population when compared with other AMD prevalence studies is the age of the individuals examined., 2,5,6,14,27 In all studies of eye disease in the elderly, the participation rate drops as the age of subjects increases, ${ }^{2,6}$ although those who have examined some participants at home report a remarkable response rate. ${ }^{2,28}$ The disadvantage of this approach is that examination conditions lose standardisation, and the use of a fundus camera, which places least reliance on subjective assessment, ${ }^{8}$ is clearly impractical. The participation rate in other prevalence studies which include individuals $>75$ years varies from $75 \%$ to $91 \%$ overall, though the rates for the most elderly participants are not specifically given. ${ }^{2-4,6,27,28}$ Nevertheless, we feel that our response rate reflects the reality of studies in this age group in the United Kingdom, although the prevalence details should clearly be interpreted with this in mind. Our assessment of the visual status of participants versus non-participants provides some reassurance, although our prevalence rates may be under-representative of the true picture of the visual morbidity of AMD in the very elderly.

The other important aspect to be considered when interpreting detailed retinal data, is the presence of media opacities. When these are severe enough to render drusen ungradable, it is possible to quantify their impact; however, milder grades of lens opacities have a variable effect on visibility of fine retinal detail, which should be borne in mind when interpreting such data in this age group. In our study this problem may artefactually suggest a lower prevalence of the more subtle lesions at the subsequent examination.

The prevalence of definite drusen in this cohort study was $72.8 \%$ initially (when aged $77-90$ years), and $62.7 \%$ subsequently (when aged $84-97$ years). This contrasts with the figure of $92.2 \%$ per person (82.7\% in right eyes) in 75- to 86-year-olds found by Klein et al. ${ }^{6}$ using the Wisconsin Grading System. One may speculate that the explanation of this difference concerns the visibility of the more subtle drusen. Firstly, the Kelvin rating of our viewing box was unknown and may have been different from that used by Klein et al., ${ }^{24}$ possibly rendering subtle drusen less visible. Secondly, the prevalence of lens opacities, or more importantly aphakia, may have differed between the two studies. If in our study we include drusen graded as questionable or indistinct, then the overall prevalence figure for drusen rises to $86.7 \%$ when the cohort was aged $77-90$ years and $74.7 \%$ when aged $84-97$ years. By contrast, Vinding ${ }^{5}$ found the prevalence of drusen in 75- to 80-year-olds to be only $32.8 \%$, although the precise thresholds for recording drusen were not given.
Our data on the largest drusen present, the most common type present, and the type occupying the largest area, give a composite picture of the interrelationships of the various drusen characteristics in an eye. It is clear that the prevalence of reticular drusen rises with age, whilst the prevalence of small (hard) and larger (soft) drusen as the predominant drusen type, falls with age. Klein et al. ${ }^{6}$ also note a fall in the frequency of small drusen as the largest size present, although they comment that the prevalence of soft drusen rises with age. The observed fluidity of these features is compatible with the recent work of Sarks et al. ${ }^{12}$ on the evolution of drusen. These authors present evidence of small hard drusen fusing to become hard clusters, which may in turn develop into soft drusen. Eventually the regression of these soft drusen may be associated with focal atrophic AMD. These changes may appear faster and to a greater extent closer to the fovea and, although the overall numbers of hard drusen need not necessarily fall as those of soft drusen rise, the present study, as well as that of Klein et al., ${ }^{6}$ support this changing relationship.

Sarks et al. ${ }^{12}$ have distinguished a group of soft drusen of a different composition. These appear to be derived from membraneous debris rather than resulting from the fusion of existing hard drusen. They found these soft drusen to be associated with diffuse pathology of the RPE, widespread basal laminar deposit and subretinal neovascular membranes. Their description of the clinical appearance of these particular soft drusen may be similar to the 'soft indistinct' category in the Wisconsin grading system, ${ }^{24}$ although further studies are required to define clearly what may become an important prognostic distinction. The Wisconsin system defined distinct and indistinct soft drusen partly on the basis of stereoscopic features, which were not available in this study. Using non-stereoscopic characteristics, a small number of soft indistinct drusen were identified, but for reasons both of small numbers and the lack of stereopsis, distinct and indistinct soft drusen were amalgamated for analyses. In this context it is interesting to note that despite a large number of eyes in this study showing other evidence of diffuse RPE changes, the number with clinically apparent neovascular membranes remained very small.

The prevalence of drusen confluence has been noted to increase with age. ${ }^{6,21,29}$ Taking the total confluence figures for the initial examination (age 77-90 years) and for the subsequent examination (age 84-97 years), this study appears to confirm an association between increased confluence and older age in an unselected population. The breakdown by age within the initial and subsequent examinations, however, does not support this observation, although the numbers of affected eyes within the age strata are 
small. Bressler and co-workers, ${ }^{21}$ examining a hospital-based population aged $44-88$ years with either bilateral drusen, or unilateral exudative disease and drusen in the fellow eye, found confluent drusen in $26.3 \%$ and $55.1 \%$ respectively. The fact that their prevalence rates are broadly similar, but in a younger age group, is likely to be due to the hospital-based nature of their sample.

Reticular drusen, which by definition represent the most confluent class of drusen in this grading system, increase in prevalence with both age and time. Sarks et al. ${ }^{12}$ have stated that on histological examination they could find no extracellular material to correspond to this lobular clinical appearance, and attribute it to vascular insufficiency in the choroid, similar to that found in pre-eclampsia. ${ }^{30}$ In AMD there are vascular changes in the choroid, with loss of capillary fenestration, fibroblast infiltration, and eventual loss of the choriocapillaris exposing larger whiter vessels beneath. ${ }^{20}$ There is good evidence that this change is secondary to loss of RPE cells and photoreceptors ${ }^{20}$ and if 'reticular drusen' are indeed areas of abnormal choroidal vasculature, then their presence would signify loss of RPE (and photoreceptors) in these areas. As a casual observation during the course of our study, we occasionally noted distinct drusen apparently overlying a paler reticular pattern. Tso ${ }^{31}$ has shown that drusen distribution bears some relationship to the lobular pattern of the underlying choroidal vessels, an observation which may support Sarks' hypothesis regarding the nature of 'reticular drusen'. Further studies are required to clarify this point, but current evidence suggests that 'reticular drusen' may indicate significant disease of the RPE.

The RPE undoubtedly has a central role in the pathogenesis of AMD,${ }^{17,20,32.33}$ and yet most clinical studies make little mention of the clinical changes in the RPE in AMD, with the occasional exception of increased pigmentation. RPE degeneration has a spectrum of appearances. These include subtle patchy pallor, tiny greyish areas suggestive of faded drusen, and marked atrophic change, although without the very abrupt margins and clearly visible choroidal vasculature that characterise geographic atrophy. Geographic atrophy, which represents the most extreme form of RPE degeneration, is scored separately by this grading system. Klein et al. ${ }^{6}$ found a much lower prevalence of RPE degeneration in their sample of 43 - to 84 -year-olds $(8.3 \%)$, but this did rise dramatically to $20.7 \%$ in the over- $75 \mathrm{~s}$. This dramatic increase with age appears to be borne out by the data presented here. On the other hand, this study uniformly found a much lower prevalence of increased pigmentation than the $25 \%$ noted by Klein et al. for those aged over 75 years. The reasons for this apparent discrepancy are not obvious, but as with drusen, differences in the visibility of the fundus may have played a role.

As with geographic atrophy, the prevalence of exudative AMD is fortunately low in this elderly population, even at subsequent examination (3.2\%). This rate is comparable with that of the Framingham study $(1.5 \%$ in persons $>52$ years $)$, the study by Vinding (1.9\% amongst 60 - to 80 -year-olds), and that by Klein et al. $(6.7 \%$ of females and $2.6 \%$ of males, over 75 years). ${ }^{5,6,27}$ All figures are likely to underrepresent the true prevalence of severe AMD, but for reasons of non-participation rather than visibility, as these lesions are relatively easily visualised.

The prevalence of any AMD per eye (at least one definite feature) in this population was $82.9 \%$ at age $77-90$ years, and $85.4 \%$ at age $84-97$ years. Although this study presents prevalence data per eye, the most prevalent feature of AMD, namely drusen, demonstrate a high level of concordance between right and left eye. ${ }^{16,33}$ Comparison between our Table XI (all eyes) and Table XII (patient's eye with the better acuity) suggest that our 'by eye' data probably represent a reasonable approximation to prevalence rates per person. Our prevalence rates for any AMD (both by eye and by person for the better eye) ranged between $82 \%$ and $86 \%$, which was considerably higher than that found in the Beaver Dam study (36.8\% in persons $>75$ years), the Framingham study (50\% in persons $75-85$ years) and the Danish study (45.1\% in persons $75-85$ years). ${ }^{6,28,24}$ The very different protocols used in the Framingham and Danish studies may explain their much lower prevalence rates; however, the present study used the same protocol as the Beaver Dam study, and the difference in rates may be partly due to the definition of 'any AMD'. In defining 'any AMD', the Beaver Dam study excluded eyes with only soft distinct or hard drusen, unless these were accompanied by RPE degeneration, increased pigmentation or other features of AMD. The present study has included such eyes in the definition of 'any AMD'.

The 'by eye' prevalence rate of any AMD in association with visual impairment (6/9 or worse) was $81.8 \%$ initially and $86.6 \%$ at follow-up. These figures are also considerably higher than those reported by others, in 75 - to 85 -year-olds, of $9.1-28 \% .^{2,13,28}$ This difference is again at least partly due to differing protocols.

The prevalence of blindness in eyes reported here ( $10.1 \%$ initially, age $77-90$ years; and $20.9 \%$ subsequently, age 84-97 years) is also significantly higher than that found by most other studies of $1.7-2.7 \%$ at $>75$ years. ${ }^{6,13,28}$ The explanation may lie in the very elderly age structure of the present study. Martinez et al. ${ }^{2}$ have shown the prevalence of blindness to increase dramatically between ages 80,85 and 90 years. Indeed, Martinez et al. ${ }^{2}$ found similar high 
rates of blindness per person $(12 \%>75$ years, and $20 \%>85$ years). This is of particular interest, as that report included a number of individuals in residential care, where the prevalence of blindness is likely to be much higher. ${ }^{35}$ Although the acuity testing of some of those residents lacked standardisation, the present study, with a much lower participation rate, found the prevalence of blindness per person to be $4.9 \%$ at age $77-90$ years, and $14.6 \%$ at age $84-97$ years results which are similar to those of Martinez et al. With current demographic shifts towards an increasingly elderly population, our study suggests that the number of elderly people with a severe visual handicap can be expected to rise dramatically.

\section{CONCLUSION}

This 'double' prevalence study provides detailed documentation of the prevalence of a variety of AMD features in a population-based sample of individuals at two points in time (aged 77+ and 84+ years). Our data confirm that in the United Kingdom AMD is an important cause of visual morbidity with a high population prevalence amongst the elderly.

The authors wish to thank the Latham House Medical Practitioners for allowing us to examine their patients, Professor Michael Clarke and the Department of Epidemiology and Public Health of the University of Leicester for population and survivorship information, Hugh Harris for photographic assistance, and Kath Robinson and Rachel Gowing for clerical assistance. This study was supported in part by the Anne Allerton fund and by the National Eye Research Council.

Key words: Age-related macular degeneration, Prevalence, Wisconsin grading.

\section{REFERENCES}

1. European Union: Demographic statistics: Luxembourg: Eurostat, 1991.

2. Martinez GS, Campbell AJ, Reinken J, Allan BC. Prevalence of ocular disease in a population study of subjects 65 years old and older. Am J Ophthalmol 1982;94:181-9.

3. Haakinen L. Vision in the elderly and its use in the social environment. Scand J Soc Med 1984;35(Suppl): $5-60$.

4. Gibson JM, Rosenthal AR, Lavery J. A study of the prevalence of eye disease in the elderly in a community. Trans Ophthalmol Soc UK 1985;104:196-203.

5. Vinding T. Occurrence of drusen, pigmentary changes and exudative changes in the macula with reference to age-related macular degeneration: an epidemiological study of 1000 aged individuals. Acta Ophthalmol (Copenh) 1990;68:410-4.

6. Klein R, Klein BEK, Linton KLP. Prevalence of agerelated maculopathy. The Beaver Dam Eye Study. Ophthalmology 1992;99:933-43.

7. Annual abstract of statistics. Central Statistical Office. London: HMSO, 1994:130.

8. Ederer F. Methodological problems in eye disease epidemiology. Epidemiol Rev 1983;5:51-66.
9. Bressler NM, Bressler SB, Fine SL. Age-related macular degeneration. Surv Ophthalmol 1988;32: 375-413.

10. Ferris FL. Senile macular degeneration: review of epidemiologic features. Am J Epidemiol 1983; 118:132-51.

11. Sarks SH. Ageing and degeneration in the macular region: a clinico-pathological study. $\mathrm{Br} \mathrm{J}$ Ophthalmol 1976;60:324-41.

12. Sarks JP, Sarks SH, Killingsworth MC. Evolution of soft drusen in age-related macular degeneration. Eye 1994;8:269-83.

13. Kahn HA, Leibowitz HM, Ganley JP, et al. The Framingham Eye Study. I. Outline and major prevalence findings. Am J Epidemiol 1977;106:17-32.

14. Lui IY, White L, LaCroix AZ. The association of agerelated macular degeneration and lens opacities in the aged. Am J Public Health 1989;79:765-9.

15. Cheraskin E. Macular degeneration: how big is the problem? J Natl Med Assoc 1992;84:873-6.

16. Coffey AJH, Brownstein S. The prevalence of macular drusen in postmortem eyes. Am J Ophthalmol 1986; 102:164-71.

17. van der Schaft TL, Mooy CM, de Bruijn WC, Oron FG, Mulder PG, de Jong PT. Histological features of the early stages of age-related macular degeneration: a statistical analysis. Ophthalmology 1992;99:278-86.

18. Sparrow JM, Dickinson AJ, Duke AM, Thompson JR, Gibson JM, Rosenthal AR. Seven-year follow-up of age-related macular disease in an elderly British population. Eye 1997;11:315-24.

19. Sarks SH. Drusen and their relationship to senile macular degeneration. Aust J Ophthalmol 1980;8: 117-30.

20. Sarks JP, Sarks SH, Killingsworth MC. Evolution of geographic atrophy of the retinal pigment epithelium. Eye 1988;2:552-7.

21. Bressler NM, Bressler SB, Seddon JM, Gragoudas ES, Jacobson LP. Drusen characteristics in patients with exudative versus non-exudative age-related macular degeneration. Retina 1988;8:109-14.

22. Pauleikhoff D, Barondes MJ, Minassian D, Chisholm $\mathrm{IH}$, Bird AC. Drusen as risk factors in age-related macular disease. Am J Ophthalmol 1990;109:38-43.

23. The International ARM Epidemiological Study Group. An international classification and grading system for age-related maculopathy and age-related macular degeneration. Surv Ophthalmol 1995;39:367-74.

24. Klein R, Davis MD, Magli YL, Segal P, Klein BE, Hubbard L. The Wisconsin age-related maculopathy grading system. Ophthalmology 1991;98:1128-34.

25. Sparrow JM, Dickinson AJ, Duke AM. The Wisconsin Age-related Macular Degeneration Grading System: performance in an independent centre. Ophthalmic Epidemiol 1997;4:49-55.

26. Clarke M, Clarke SM, Odell A, Jagger C. The elderly at home: health and social status. Health Trends 1984;16:3-7.

27. Leibowitz HM, Krueger DE, Maunder LR, et al. The Framingham Eye Study Monograph. Surv Ophthalmol 1980;24(Suppl):335-610.

28. Vinding T. Age-related macular degeneration: macular changes, prevalence and sex ratio. An epidemiological study of 100 aged individuals. Acta Ophthalmol (Copenh) 1989;67:609-16.

29. Smiddy WE, Fine SL. Prognosis of patients with bilateral macular drusen. Ophthalmology 1984;91: 271-7. 
30. Saito Y, Omoto T, Fukuda M. Lobular pattern of choriocapillaris in pre-eclampsia with aldosteronism. Br J Ophthalmol 1990;74:702-3.

31. Tso MOM. Pathogenetic factors of ageing macular degeneration. Ophthalmology 1985;92:628-35.

32. Dorey CK, Wu G, Ebenstein D, Garsd A, Weiter JJ. Cell loss in the ageing retina: relationship to lipofuscin accumulation and macular degeneration. Invest Ophthalmol Vis Sci 1989;30:1691-9.
33. Barondes MJ, Pauleikhoff D, Chisholm IC, Minassian $\mathrm{D}$, Bird AC. Bilaterality of drusen. Br J Ophthalmol 1990;74:180-2.

34. Sperduto RD, Seigel D. Senile lens and senile macular changes in a population-based sample. Am J Ophthalmol 1980;90:86-91.

35. Klein R, Klein BEK, Linton KLP, De Mets DL. The Beaver Dam Eye Study: visual acuity. Ophthalmology 1991;98:1310-5. 